\title{
TWO-DIMENSIONAL HYDRODYNAMICAL SIMULATIONS \\ OF WIND-COMPRESSED DISKS \\ AROUND RAPIDLY ROTATING B-STARS
}

\author{
S. P. OWOCKI and S. R. CRANMER \\ Bartol Research Institute, University of Delaware, \\ Newark, DE 19716, U.S.A. \\ and \\ J. M. BLONDIN \\ Department of Physics, North Carolina State University, \\ Raleigh, NC 27695, U.S.A.
}

We use a 2-D PPM code to simulate numerically the hydrodynamics of a radiation-driven stellar wind from a rapidly rotating Be-star. The results generally confirm predictions of the semi-analytic "Wind Compressed Disk" model recently proposed by Bjorkman and Cassinelli to explain the circumstellar disks inferred observationally to exist around such rapidly rotating stars. However, this numerical simulation is able to incorporate several important effects not accounted for in the simple model, including a dynamical treatment of the outward radiative driving and gas pressure, as well as a rotationally oblated stellar surface. This enables us to model quantitatively the compressed wind and shock that forms the equatorial disk. The simulation results thus do differ in several important details from the simple model, showing, for example, cases of inner disk inflow not possible in the heuristic approach of assuming a fixed outward velocity law. In addition, the disk opening typically has a half-angle of 2-4 degrees, somewhat larger than the $\sim 0.5^{\circ}$ predicted from the analytic model, and there is no evidence for the predicted detachment of the disk that arises in the fixed outflow picture.

Despite these differences, if the radial velocity in the analytic model is fixed to be the same as that found in the dynamical simulation, then the analytically derived equatorward flow that characterizes the equatorial focusing effect is in excellent agreement with that in the fully dynamical model. Hence, the general predicted effect of disk formation by wind focussing toward the equator is substantially confirmed.

This paper has been accepted for publication in the April 1, 1994 issue of the Astrophysical Journal. 